\title{
Körperorientierte Intervention im Coaching
}

\author{
Natascha Lienhard • Hansjörg Künzli
}

Eingegangen: 6. Dezember 2020 / Angenommen: 18. Dezember 2020 / Online publiziert: 3. Februar 2021 (C) Der/die Autor(en) 2021

Zusammenfassung In der Coaching-Literatur sind körperorientierte Interventionen untervertreten oder haben nicht den Stellenwert, der ihnen zukommen könnte. Zu der Frage, ob und in welchem Ausmaß sich dies auch in der Coachingpraxis spiegelt, führten wir im Herbst 2020 eine Online-Umfrage durch. Insgesamt 242 Coaches beantworteten 45 Fragen zu vier Themenkreisen körperorientierter Interventionen. Die meisten der befragten Coaches setzen körperorientierte Interventionen ein, betrachten sie als zielführend und sinnvoll für Coaching und wollen sie in Zukunft gleich oft oder noch intensiver einzusetzen. Große Unterschiede sind festzustellen hinsichtlich der Art der eingesetzten Interventionen. Körperhaltung und Mimik wird von fast allen Coaches als Erkenntnisquelle genutzt. Die aktive Arbeit mit dem Körper, z. B. in Form von Bewegungsübungen, wird weniger oft eingesetzt. Das Thema Körperorientierte Intervention im Coaching ist noch weitgehend unerforscht. Ebenso entsteht der Eindruck, dass der Begriff „,körperorientierte Intervention“ im Coaching noch sehr unterschiedlich gedeutet wird.

Schlüsselwörter Coaching $\cdot$ Körperorientierte Intervention · Online-Umfrage

N. Lienhard, BSc $(\bowtie) \cdot$ Prof. H. Künzli (夰)

Departement Psychologie/Psychologisches Institut, ZHAW, Pfingstweidstrasse 96, 8037 Zürich,

Schweiz

E-Mail: liea@zhaw.ch; kasg@zhaw.ch 


\title{
Body-oriented interventions in coaching
}

\begin{abstract}
In the coaching literature, body-oriented interventions are underrepresented or do not have the importance they could have. To approach the question whether and to what extent this situation is also reflected in coaching practice., we conducted an online survey in the fall of 2020. A total of 242 coaches responded to 45 questions on the four themes of body-orientated interventions. The vast majority of coaches surveyed use body-oriented interventions, they consider them purposeful and useful for coaching, and they express the intention to use them as often or even more intensively in the future. Large differences can be observed with regard to the type of interventions used. Body posture and facial expressions are used by almost all coaches as a source of insight. Active work with the body, e.g. in the form of movement exercises, is used less often. The topic of body-oriented intervention in coaching is still largely unexplored. Likewise, the impression arises that the term "body-oriented intervention" in coaching is still interpreted very differently.
\end{abstract}

Keywords Coaching · Body-oriented interventions · Online survey

\section{Einleitung}

„Wenn man den Körper grad zufällig dabeihat, dann könnte man ihn ja auch grad nutzen." So oder ähnlich leitete Gunther Schmidt im Laufe seiner Coaching-Ausbildung manchmal körperorientierte Interventionen ein. Die Art der Einleitung verweist, neben dem offensichtlichen Inhalt der Aussage, dass man den Körper eben auch im Coaching nutzen kann, auf mindestens einen Subtext: auf eine gewisse Beiläufigkeit von körperorientierten Interventionen im Coaching. Körperorientierte Interventionen stehen im Coaching nicht im Zentrum des Interesses, sondern kommen zum Zuge, wenn es ,grad so passt“. Auf der einen Seite ist dies erstaunlich, erlebt doch der „Körper“ in der Arbeitswelt eine wahre Renaissance. Immer mehr Unternehmen bieten, z. B. im Rahmen des Gesundheitsmanagements, Achtsamkeitstrainings, Yoga, Entspannungsübungen und weitere körperorientierte Maßnahmen an. Die Aufrechterhaltung der körperlichen Gesundheit ist nicht mehr nur Privatsache, sondern strategische Aufgabe der Organisation geworden.

Von daher ist es unwahrscheinlich, dass Coaching von dieser Entwicklung nicht betroffen sein sollte. Und tatsächlich, gibt man den Begriff ,körperorientiertes Coaching“ in Google ein, resultieren über 122.000 Einträge. In der Praxis scheint die Körperorientierung im Coaching angekommen zu sein. Anders sieht es in der einschlägigen Literatur zum Coaching aus. Dort führt der Körper ein Mauerblümchendasein. Zwar gibt es kaum ein Coaching-Lehrbuch, in dem nicht der Körper als Informations- und Erkenntnisquelle und/oder Resonanzraum dargestellt wird. Artikel oder Werke, deren Gegenstand allein der Körper im Coaching ist, findet man aber eher selten. Als Ausnahmen sind hier sicher die Artikel von Pesendorfer und Pechtl (2018), Ryba (2019), Pullen (2016) oder das Buch zum Embodiment von Storch et al. (2017) zu nennen. Woran es liegt, dass die theoretische Beschäftigung mit der Praxis nicht Schritt halten kann, ist unklar. Ein Grund mag sein, dass die 
im deutschen Sprachraum am stärksten verbreiteten Ansätze des lösungs- oder des systemisch-lösungsorientierten Coachings (Seiger und Künzli 2012; Hofmann und Künzli 2021) den Körper zwar keineswegs negieren, aber stark auf die Sprache, z. B. in Form von unterschiedlichen Fragetypen, fokussieren.

Doch was ist körperorientiertes Coaching? Eine einheitliche Definition scheint es nicht zu geben, obwohl viele Coaches ,körperorientiertes Coaching“ anbieten. Auch in der Körperpsychotherapie steht eine umfassende Definition noch aus. Betrachtet man verschiedene Definitionen, sind hingegen Konvergenzen festzustellen.

So unterschiedlich die verschiedenen Definitionen und Beschreibungen körperorientierter Arbeit in der Psychotherapie und im Coaching auch sind, beinhalten sie doch meistens die folgenden vier Komponenten: (1) Körper und Psyche bilden eine untrennbare Einheit (Ryba 2019; Storch et al. 2017; Pullen 2016); (2) Ziel der körperorientierenten Arbeit ist das Selbsterleben und der Zugang zum Unbewussten (Ryba und Roth 2019; Geuter 2019); (3) es sind sehr verschiedene Zugänge zur Körperwahrnehmung und zum Körpererleben möglich (z. B. Geuter 2019; Schatz 2002); und (4) die körperorientierte Arbeit in Therapie und Coaching wird von der rein manuellen Körperarbeit abgegrenzt (z. B. Geuter 2019; Schley 2015).

Was alle diese Autoren einstimmig betonen, ist die Einheit von Körper und Psyche (Leib/Seele). Beide können als Zugang zum inneren Erleben genutzt werden. So betonen z. B. Storch et al. (2017) für das Konzept des Embodiment (,,Verkörperung“) „dass der Geist (also: Verstand, Denken, das kognitive System, die Psyche) mitsamt seinem Organ, dem Gehirn, immer in Bezug zum gesamten Körper steht. Geist/ Gehirn und Körper wiederum sind in die restliche Umwelt eingebettet.“

Wie Geuter (2019) sieht auch Ryba (2019) die Zielsetzung körperorientierter Interventionen im Coaching im Zugang zum Unbewussten und in einem veränderten Selbsterleben. Als besonders bedeutsam für ein nachhaltiges Coaching nennt sie die Aspekte des willkürlichen und des unwillkürlichen Bewegungsausdrucks, der Körperwahrnehmung und -kommunikation, der Körperarbeit und der Veränderung von Schemata. Eine differenziertere Betrachtung der Zielsetzung stammt aus der Psychotherapie. Affektorientierte Schulen der Psychotherapie (z.B. Bioenergetik oder Biodynamik) haben laut Geuter (2019) u. a. als Ziel, muskuläre Verspannungen zu lösen, welche den Zugang zum Innerpsychischen blockieren. So können verdrängte Gefühle mobilisiert und zur Verarbeitung zugänglich gemacht werden. Wahrnehmungsorientierte Schulen (u. a. Konzentrative Bewegungstherapie oder Funktionelle Entspannung) verfolgen das Ziel, dass ihre Klientinnen und Klienten sich körperlich selbst erfahren, also eine Art körperorientierte Achtsamkeit entwickeln und damit experimentieren. Implizites Erleben, wenn auch teilweise schwer verbalisierbar, kann so deutlich gemacht werden. Beziehungsorientierte Schulen wie z. B. Analytische Körperpsychotherapie lassen innere Objektrepräsentanzen körperlich (z.B. Rollenspiele, Körperausdruck) externalisieren, um korrigierende Erfahrungen zu erleben. Zuletzt gibt es noch Bewegungsorientierte Schulen wie z. B. die Tanztherapie. Diese versuchen, mittels des Ausdrucks inneren Erlebens in Bewegung den Kontakt zu anderen und zu sich selbst zu verbessern (Ryba 2019).

Die Zugänge, Methoden und konkreten Techniken, mit denen gearbeitet wird, können sehr unterschiedlich und vielfältig sein. Unter körperorientierter Arbeit, die das Selbsterleben und den Zugang zum Unbewussten unterstützen, zählt Ryba (2019) 
u.a. PMR, autogenes Training, Meditation, fokussierte Aufmerksamkeit oder auch hypnotherapeutische Trance auf.

Wichtig erscheint uns, auf das Abgrenzungskriterium zwischen körperorientierten Psychotherapien und körperorientiertem Coaching im Vergleich zur Ausführung einer rein körperlichen Ausführung einer Anleitung, wie dies z. B. in der Physiotherapie praktiziert wird, hinzuweisen. Geuter (2019) betont, dass oft in der Körperpsychotherapie die „Körperarbeit“ oder „,body work“ als Abgrenzungsmerkmal zu anderen, rein sprachbasierten Therapieformen gesehen werde. Diese Verwendung des Begriffs wird von Geuter (2019) kritisiert, da der Körper lediglich den Zugang zum Selbsterleben darstelle, welches aber ohne Kognition nicht erlebbar oder beschreibbar wäre. Schley (2015) merkt außerdem an, dass der Begriff „Körperarbeit““ dem Geschehen in der Therapie nicht gerecht werde, da auch mittels Körperarbeit ein Dialog entstehe und nicht nur eine körperliche Ausführung anhand einer Instruktion eines/einer Therapeut/in. Ähnlich argumentiert Geuter (2019). Er versteht die Arbeit mit dem Körper als Wechsel vom Sprechen alleine zu ,einer Arbeitsweise, die Bewegung, Atmung oder körperlichen Ausdruck einbezieht“". Beiden Autoren ist wichtig, dass es sich bei der Körperarbeit um eine Erweiterung und Vertiefung des therapeutischen Dialogs handle und nicht um einen per se neuen Zugang. Um dem Rechnung zu tragen, sprechen wir in der vorliegenden Arbeit von körperorientiertem Coaching oder körperorientierter Arbeit im Coaching. Damit wollen wir betonen, dass körperorientierte Arbeit als Unterstützung eines herkömmlichen Coachings eingesetzt wird.

Aus diesen Erwägungen haben wir versucht, eine möglichst generische Arbeitsdefinition körperorientierter Interventionen im Coaching für die vorliegende Studie abzuleiten, die von den vielen Schulen, Zugängen, Methoden und Techniken abstrahiert, das Feld aber trotzdem angemessen abdeckt. Die Arbeitsdefinition enthält drei der vier o.g. Komponenten. Die Annahme der Einheit von Körper und Geist bzw. Leib und Seele wurde weggelassen, da die Dualitätshypothese (der Körper wirkt auf den Geist und umgekehrt) nach wie vor oft verwendet wird und die Abgrenzung für unsere Zwecke keine Rolle spielt:

Unter körperorientiertem Coaching werden in dieser Studie Methoden verstanden, welche den Körper verbal, visuell oder sensorisch in den Fokus der Aufmerksamkeit der Coachees rücken, um innere Prozesse bewusst zu machen oder auszulösen. Rein manuelle Techniken, wie z. B. kraniosacrale Therapie, klassische Massagen oder körperliche Betätigungen wie Dehnungsübungen zählen wir hier nicht dazu, da dort nicht auf das Erleben und die Reflexion innerpsychischer Prozesse, ausgelöst durch die Körperübung, fokussiert wird.

In der einschlägigen Coaching-Literatur ist der Körper untervertreten oder hat zumindest nicht den Stellenwert, der ihm zukommen könnte. Unklar bleibt, ob und in welchem Ausmaß sich dieser Sachverhalt auch in der Coachingpraxis spiegelt. Um uns dieser Frage anzunähern, haben wir im Herbst 2020 eine Umfrage zu diesem Thema durchgeführt. Die Resultate sollen einen ersten Einblick geben, ob und wie Coaches den Körper in ihre Arbeit integrieren. Konkret lautete die Fragestellung: Welche Arten von körperorientierten Methoden wenden Coaches an, wie häufig und in welcher Intensität kommen diese Methoden zum Einsatz und welche Bedeutung 
messen sie solchen Interventionen in ihrer gegenwärtigen und zukünftigen CoachingPraxis bei?

\section{Methode}

Stichprobe Die Umfrage wurde von 242 deutschsprachigen Coaches ausgefüllt, welche durch den Berufsverband Supervision Organisationsberatung und Coaching BSO und das IAP via E-Mail oder Newsletter verbreitet wurde. Im Schnitt $(N=223)$ waren die Teilnehmenden $53(M=53,41, S D=7,89)$ Jahre alt, wobei die jüngste 30 und die älteste Person 73 Jahre alt war. Teilgenommen haben 158 Frauen $(71 \%)$ und 64 Männern (29\%), 19 Personen machten keine Angaben, und eine Person gab „Anderes“ als Geschlecht an. Über eine Habilitation verfügen 1,2\%, 4,1\% über eine Promotion, 56,2 \% über einen Masterabschluss auf Universitäts- oder Fachhochschulstufe, 15,7\% über einen Bachelor auf Universitäts-, oder Fachhochschulstufe, 15,7\% über einen Abschluss auf Bachelorstufe und 1,2\% über eine gymnasiale Matura, eine Fachmittelschul-/Handelsmittelschulabschluss. 3,7\% nannten einen anderen Ausbildungsabschluss.

Coachingausbildung 151 (62\%) Teilnehmende absolvierten einen Master of Advanced Studies MAS oder wiesen eine vom bso anerkannte (bso 2019) Ausbildung im Bereich Coaching, Supervision oder Organisationsentwicklung aus. Das am häufigsten genannte Ausbildungsinstitut war das IAP, andere oft genannte sind die FHNW, die BFH, IBP Institut, Trigon, ZAK Basel oder das Institut für systemische Impulse.

Arbeitspensum Coaching Das Arbeitspensum als Coach beträgt zwischen 40 und $50 \%$ in Bezug auf $100 \%$ Vollzeitpensum. Das Pensum der Mehrheit der Befragten (53\%) liegt zwischen 0 und $40 \%$, ein Drittel aller Befragten arbeitet zwischen 10 und $30 \%$ als Coach. Eine Minderheit (9,1\%) arbeitet zwischen 90 und $100 \%$ in ihrer Tätigkeit als Coaches.

Anzahl Jahre Erfahrung als Coach Der Durchschnitt der Coaching-Erfahrungen beträgt knapp 13 Jahre $(\mathrm{M}=12,76, \mathrm{SD}=8,20)$, mit einem Minimum von einem Jahr und einem Maximum von 40 Jahren Coaching. Bei gut der Hälfte $(51,6 \%)$ der Befragten beträgt die Erfahrung zwischen einem und 10 Jahren.

Körperorientierte Weiterbildung $64(26 \%)$ der Coaches gaben an, eine körperorientierte Weiterbildung abgeschlossen zu haben. $160(66 \%)$ verneinten dies und 18 machten keine Angaben. Die Art der genannten Ausbildungen ist sehr vielfältig (Tab. 1).

Konstruktion des Erhebungsinstruments Das Instrument enthält vier Frageblöcke zu unterschiedlichen Aspekten körperorientierter Arbeit (vgl. Tab. 1): die Art und die Häufigkeit der eingesetzten Interventionen, die Art, wie diese Interventionen einge- 
Tab. 1 Körperorientierte Weiterbildungen

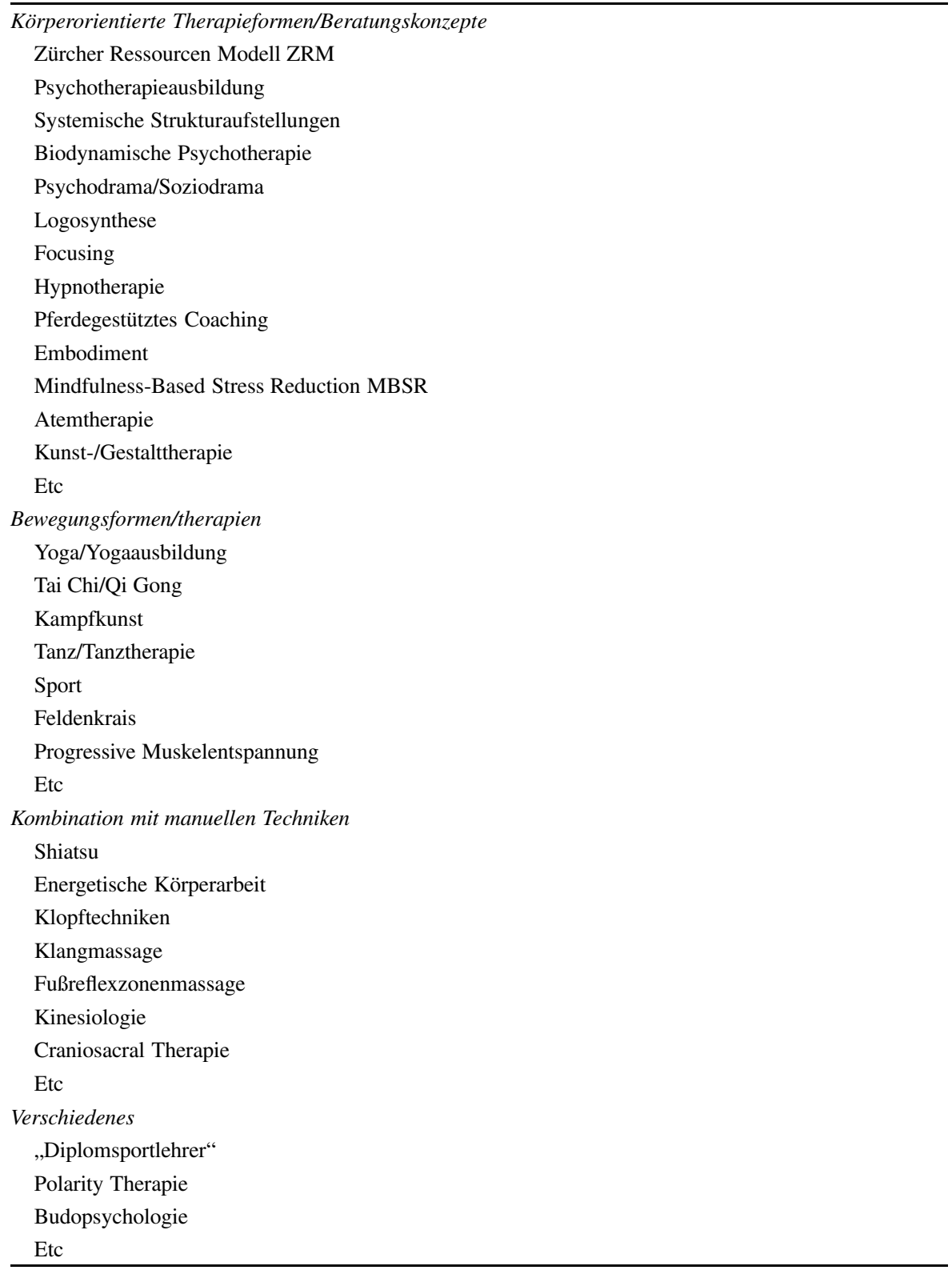

setzt werden, die Bedeutung, die Coaches körperorientierten Intervention zuweisen, und die Absicht, solche Interventionen auch in Zukunft anzuwenden.

Im Zentrum des Interesses stand die Frage nach der Art und Häufigkeit der eingesetzten Interventionen. Eine Systematisierung körperorientierter Verfahren und Techniken im Coaching existiert unseres Wissens nicht. Um der Vielfalt von prakti- 
Tab. 2 Zuordnung der Fragen zu den vier Aspekten körperorientierter Arbeit

\begin{tabular}{|c|c|c|c|}
\hline Kategorie & Frage $\mathrm{Nr}$ & Prinzip & Prozessziele \\
\hline \multirow{10}{*}{$\begin{array}{l}\text { Anwendungen von } \\
\text { körperorientierten } \\
\text { Methoden }\end{array}$} & $1.1,1.2$ & $\begin{array}{l}\text { 1. Wahrnehmen und } \\
\text { Spüren }\end{array}$ & $\begin{array}{l}\text { Grundlage zur erlebnisorientierten } \\
\text { Prozessarbeit }\end{array}$ \\
\hline & $\begin{array}{l}1.3,1.4,1.5 \\
1.6\end{array}$ & $\begin{array}{l}\text { 2. Gewahrsein und } \\
\text { Gegenwart }\end{array}$ & $\begin{array}{l}\text { Grundlage zur erlebnisorientierten } \\
\text { Prozessarbeit }\end{array}$ \\
\hline & $1.7,1.8,1.9$ & $\begin{array}{l}\text { 3. Erkunden und } \\
\text { Entdecken }\end{array}$ & $\begin{array}{l}\text { Inneres Erleben aktivieren, sondie- } \\
\text { ren }\end{array}$ \\
\hline & 1.10 & $\begin{array}{l}\text { 4. Aktivieren und } \\
\text { Ausdrücken }\end{array}$ & $\begin{array}{l}\text { Emotionales Erleben aktivieren } \\
\text { und ausdrücken }\end{array}$ \\
\hline & $\begin{array}{l}1.11,1.12 \\
1.13,1.16\end{array}$ & $\begin{array}{l}\text { 5. Regulieren und } \\
\text { Modulieren }\end{array}$ & $\begin{array}{l}\text { Dysfunktionale/Dysregulierte } \\
\text { Emotionen modulieren/regulieren }\end{array}$ \\
\hline & 1.14 & $\begin{array}{l}\text { 6. Zentrieren und } \\
\text { Erden }\end{array}$ & Autoregulation \\
\hline & & $\begin{array}{l}\text { a } 7 . \text { Berühren und } \\
\text { Halten }\end{array}$ & $\begin{array}{l}\text { Ko-regulatorische therapeutische } \\
\text { Tätigkeit }\end{array}$ \\
\hline & 1.15 & $\begin{array}{l}\text { 8. Inszenieren und } \\
\text { Interagieren }\end{array}$ & $\begin{array}{l}\text { Inneres Erleben aktivieren, sondie- } \\
\text { ren }\end{array}$ \\
\hline & $\begin{array}{l}1.17,1.18, \\
1.19\end{array}$ & $\begin{array}{l}\text { 9. Verkörpern und } \\
\text { Handeln }\end{array}$ & $\begin{array}{l}\text { Übergreifend, wichtig zur Verände- } \\
\text { rung }\end{array}$ \\
\hline & 1.20 & $\begin{array}{l}\text { 10. Reorganisieren } \\
\text { und Transformieren }\end{array}$ & $\begin{array}{l}\text { Übergreifend, wichtig zur Verände- } \\
\text { rung }\end{array}$ \\
\hline \multirow{2}{*}{$\begin{array}{l}\text { Art der Anwendung } \\
\text { von } \\
\text { körperorientierten } \\
\text { Methoden }\end{array}$} & $2.1-2.4$ & Wie? & $\begin{array}{l}\text { Anleitung, Empfehlung, Selbstpra- } \\
\text { xis während Coachings }\end{array}$ \\
\hline & $3.1-3.5$ & Wozu? & $\begin{array}{l}\text { Entspannungstechnik, Problem- } \\
\text { bewältigung, Hilfestellung für } \\
\text { Coaches }\end{array}$ \\
\hline $\begin{array}{l}\text { Bedeutung körper- } \\
\text { orientierter Metho- } \\
\text { den }\end{array}$ & 4,5 & $\begin{array}{l}\text { Wichtigkeit und } \\
\text { Nutzen }\end{array}$ & - \\
\hline $\begin{array}{l}\text { Anwendung in der } \\
\text { Zukunft }\end{array}$ & 6 & $\begin{array}{l}\text { Wunsch nach Ein- } \\
\text { satz in der Zukunft }\end{array}$ & - \\
\hline
\end{tabular}

\section{Anmerkung:}

${ }^{a}$ Das Prinzip 7 Berühren und Halten wurde nicht abgefragt, da es nicht als notwendiges Prinzip von körperpsychotherapeutischer Arbeit deklariert wird (Rolef Ben-Shahar 2014)

zierten Verfahren und Techniken einigermaßen gerecht zu werden, orientierten wir uns zur Konstruktion der Fragen zur Anwendung von körperorientierten Methoden an den 10 übergreifenden körpertherapeutischen Prinzipien von Geuter (2019; vgl. Tab. 2). Diese beinhalten nach seinen eigenen Worten ,eine Systematisierung eines prozessorientierten therapeutischen Handelns, das die Körperpsychotherapie verfahrensspezifisch anzubieten hat". Ob sie ein getreues Abbild aller körperorientierten Verfahren darstellen, bezweifelt der Autor selbst. Dies dürfte bei der Vielfalt von Verfahren auch kaum zu realisieren sein. Für unsere Zwecke erscheinen sie aber als hinreichende Integration von sehr unterschiedlichen Verfahren. Für das 7. Prinzip „Berühren und Halten“ wurden keine Fragen formuliert, da es nicht als notwendiges Prinzip körperorientierter Arbeit gilt (Rolef Ben Shahar 2014). Aus den restlichen 9 Prinzipien wurden eine bis max. vier Fragen abgeleitet, die das dahinterliegende Prinzip abbilden. Bei den einzelnen Items handelt es sich um fünfstufige Skalen mit 
zunehmenden Häufigkeiten (Beispiel-Item: Ich achte auf die Mimik meiner Coachees; , $1=$ nie“, ,2 = manchmal", , ,3= öfter", , ,4= sehr oft" und , $5=$ immer").

Die Fragen nach der Art, wie Coaches körperorientierte Interventionen einsetzen, sollen einerseits Hinweise zur konkreten Umsetzung und andererseits zum ,Wozu“ resp. zum Verwendungszweck der verwendeten Intervention geben. Unter dem Bedeutungsaspekt wird erhoben, welche Wichtigkeit Coaches körperorientierten Interventionen beimessen. Unter dem Zukunftsaspekt wird die Absicht der Coaches erfragt, ob sie planen, körperorientierte Interventionen in Zukunft häufiger, weniger häufig oder in gleichem Ausmaß wie bisher einzusetzen.

Durchführung der Umfrage Die Umfrage dauerte vom 31. August 2020 bis zum 27. Oktober 2020. Unterstützt wurde die Studie durch den Berufsverband für Coaching, Supervision und Organisationsberatung (bso) sowie durch das Institut für Angewandte Psychologie (IAP) und die Zürcher Hochschule für Angewandte Wissenschaften (ZHAW). Sie gaben uns Rückmeldungen zur Umfrage und haben durch Mailings ihre Mitglieder bzw. ehemalige Absolventen auf die Studie aufmerksam gemacht.

Um ein gemeinsames Verständnis von körperorientierten Interventionen herzustellen, wurde den Coaches die in der Einleitung entwickelte Definition körperorientierter Interventionen gezeigt. Wichtig war uns erstens die Abgrenzung zu rein manuellen Techniken und zweitens der Hinweis, dass es nicht nur um den sensorischen, sondern auch um den verbalen und visuellen Einbezug des Körpers geht.

Auswertungsmethoden Die Daten wurden deskriptiv mit SPSS 26 ausgewertet und in Form von Häufigkeitstabellen dargestellt. Die von 1 bis 5 laufenden Ausprägungen der einzelnen Fragen des ersten Aspektes suggerieren eine kontinuierliche Skala. Die verbalen Anker verweisen aber auf nicht gleichabständige Häufigkeiten, daher wurde auf die Berechnung von Mittelwerten und Standardabweichungen verzichtet. Um die Prägnanz der Resultate zu erhöhen, haben wir die Werte der Ausprägungen „öfter“, „sehr oft“ und ,immer“ in einer ersten, und „,manchmal“, „öfter“, „sehr oft" und ,immer" in einer weiteren Spalte addiert. Die Werte der ersten kumulierten Spalte interpretieren wir so, dass die genannte Art der Intervention mit einer gewissen Regelmäßigkeit ausgeführt wird und mithin zum Standardrepertoire der antwortenden Coaches gehört. Die zweite kumulierte Spalte erlaubt den Vergleich mit den Coaches, die eine bestimmte Intervention gar nie ausführen.

Auf die Analyse möglicher Zusammenhänge von Stichprobenmerkmalen wie Alter, Geschlecht, Anzahl Jahre Erfahrung als Coach und Beschäftigungsgrad als Coach mit den Resultaten wurde aus zwei Gründen verzichtet. Einerseits würde die Darstellung dieser Resultate den Rahmen dieses Artikels sprengen. Der wichtigere Grund ist aber darin zu sehen, dass unsere Stichprobe kaum Repräsentativität beanspruchen kann, die Einflüsse dieser Variablen meist recht klein sind und schon leichte Verzerrungen der Stichprobe zu falschen Ergebnissen führen können.

Zur Ergänzung der quantitativen Fragen wurden den Coachees zwei offene Fragen $\mathrm{zu}$ weiteren verwendeten Interventionsmethoden und $\mathrm{zu}$ den Zielen, die sie mit körperorientierten Intervention verfolgen, gestellt. Die Antworten wurden nach Mayring (2010) ausgewertet. Die Kategorienbildung geschah induktiv. 


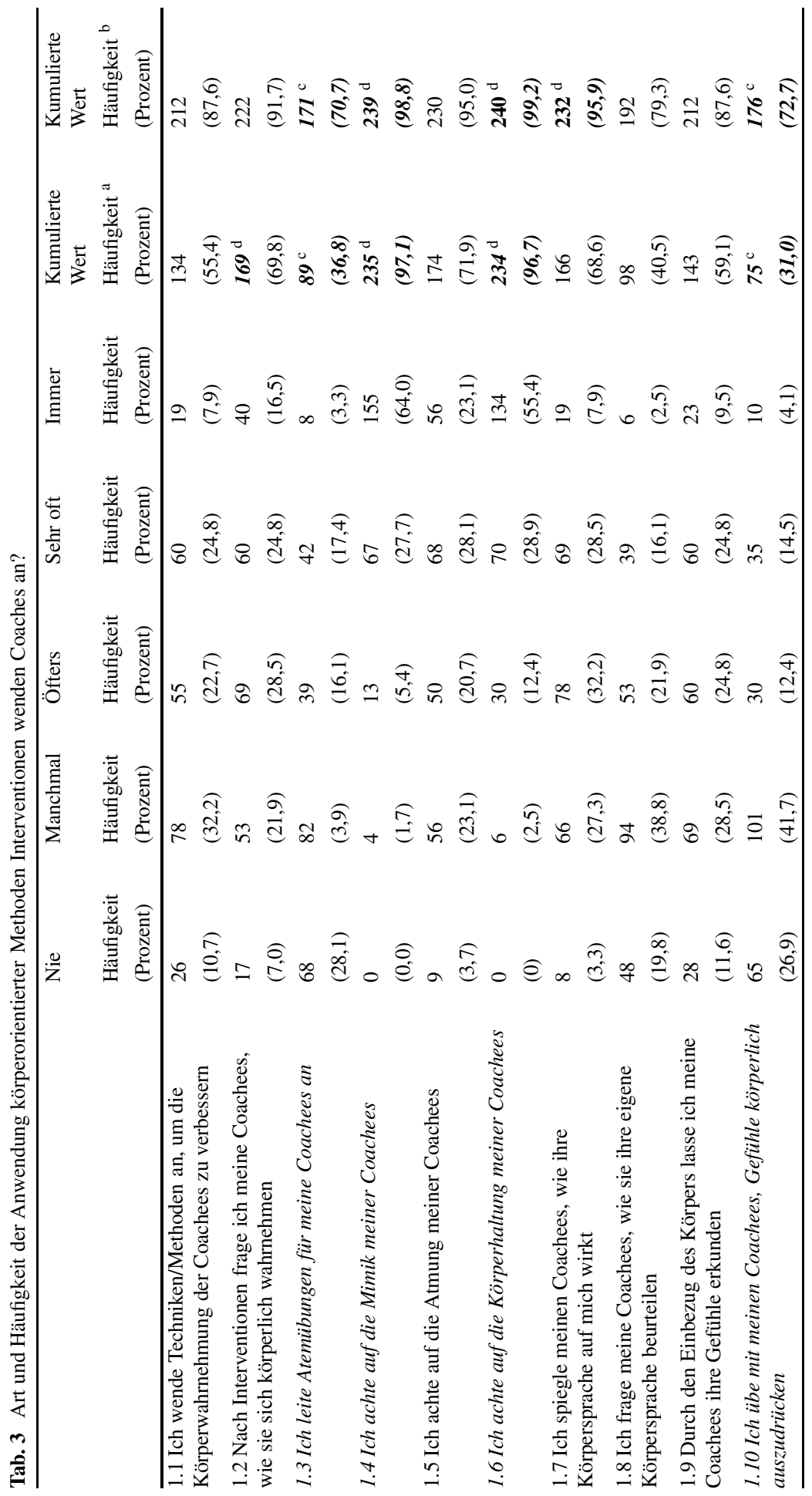




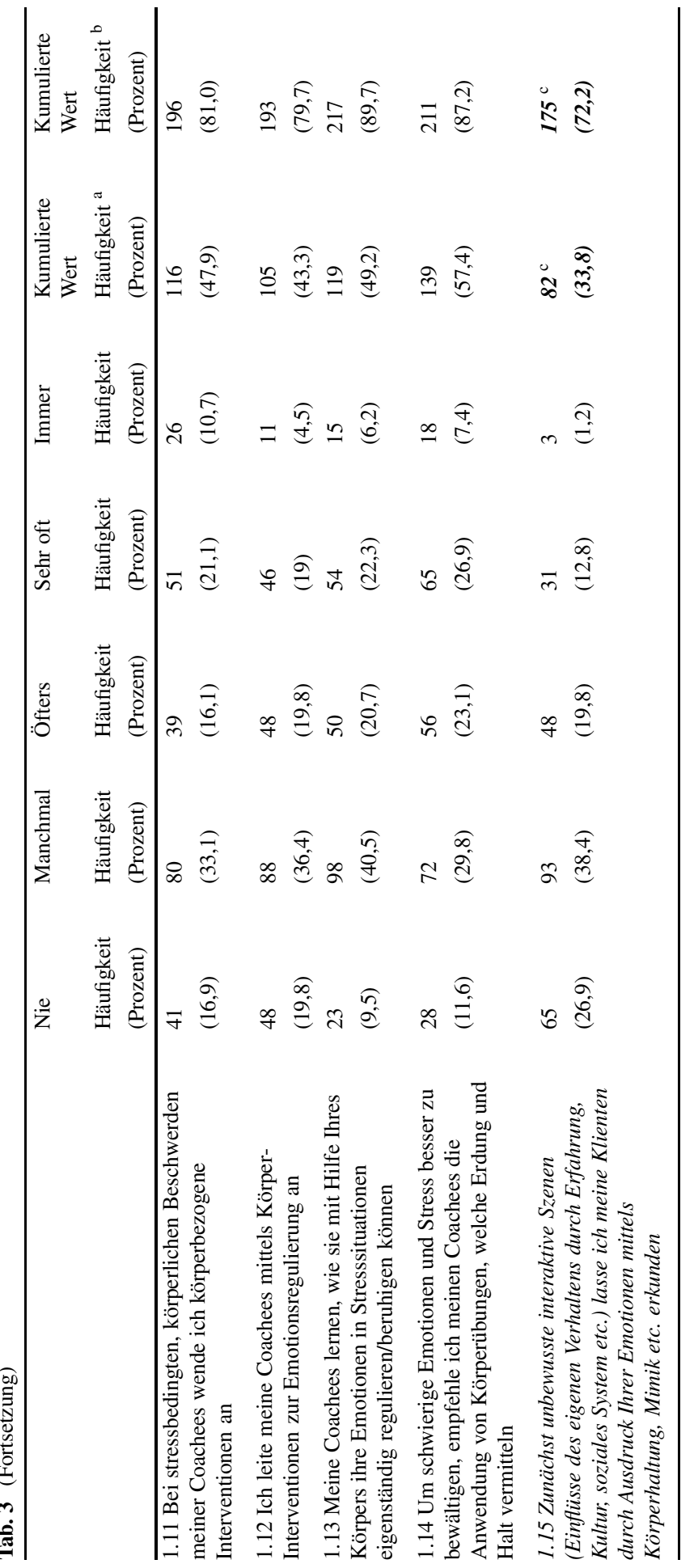




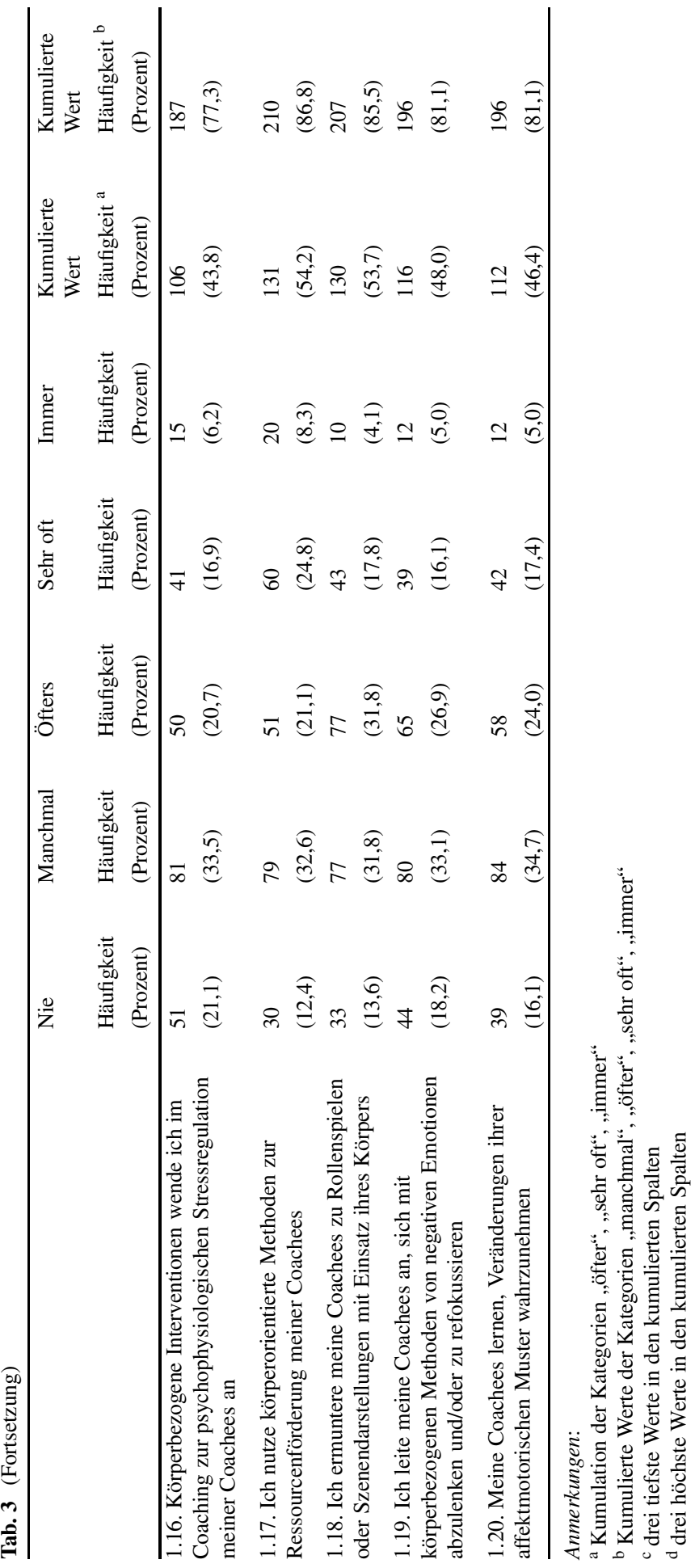




\section{Resultate}

Die Darstellung der Resultate folgt den vier Frageblöcken „Anwendung körperorientierte Interventionen“, „Art und Weise der Anwendung“, „Bedeutung, die Coaches körperorientierten Interventionen beimessen“" und "Anwendung in der Zukunft". $\mathrm{Zu}$ beachten ist, dass sich die Prozentwerte immer auf die Gesamtanzahl der Teilnehmenden von $N=242$ beziehen und sich daher aufgrund fehlender Werte nicht zwingend auf $100 \%$ aufsummieren.

In Tab. 3 werden die Art und Häufigkeit der Anwendungen von körperorientierten Interventionen dargestellt. Auffällig ist, dass die Häufigkeiten über alle Fragen recht stark streuen, praktisch keine Boden- oder Deckeneffekte festzustellen sind und dass über alle Interventionen die große Mehrheit, das heißt zwischen knapp $71 \%$ und fast $100 \%$ der Coaches angibt, die genannten Interventionen zumindest manchmal anzuwenden.

Betrachtet man die kumulierten Werte der Ausprägungen „öfter“ bis ,immer“, dann bewegen sich die Werte in einem Bereich von 31,0\% bis 97,1\%. \%. Bei praktisch allen Coaches gehört das Achten auf die Mimik (97,1\%) und die Körperhaltung $(96,7 \%)$ zum Standardrepertoire.

Tab. 4 Offene Antworten zur Art der verwendeten Intervention



Anmerkung: Basierend auf den Antworten von $n=31$ Coaches 
Die Prozentwerte in der Ausprägung ,nie“ bewegen sich zwischen 0,0 und 28,0\%. Die höchsten Prozentwerte in der Kategorie „,nie“ erreichen die Fragen zur Anleitung von Atemübungen (28\%), Übung des Gefühlsausdrucks über den Körper (26,9\%) und der szenischen Reproduktion der Gefühle über den Körper (26,9\%).

Die geringsten Werte findet man erwartungsgemäß unter der Ausprägung ,immer". Sie bewegen sich von 1,2\% für die Intervention Szenische Reproduktion über die Emotion bis zu 64,0\% für das Achten auf die Mimik. Immer noch 55,4\% geben an, immer auf die Körperhaltung zu achten.

In einer offenen Frage hatten die Coaches die Gelegenheit, anzugeben, welche Interventionen sie neben den im Fragebogen genannten sonst noch anwenden (Tab. 4). Die offenen Antworten decken sich weitgehend mit den Interventionsarten der geschlossenen Frage. Trotzdem sind noch neue Aspekte festzustellen, wie z.B. der Einsatz von Tieren, Klang und die interdisziplinäre Arbeit mit anderen Fachpersonen.

In Tab. 5 wird die Art und Weise, wie Coaches körperorientierte Interventionen einsetzen und welche Ziele sie damit verfolgen, dargestellt. Integrieren Sie die Übungen in die Coachingsitzungen? Empfehlen Sie sie Ihren Coachees zur Ausübung neben dem Coaching? Wenden Sie körperorientierte Übungen auch auf sich selbst an? Genau die Hälfte $(50,0 \%)$ geben an, Körperarbeit im Coaching einzusetzen. Hier zeigt sich ein Widerspruch zu den Resultaten in Tab. 3. Dieser Widerspruch wird in

Tab. 5 Art und Weise der Anwendung körperorientierter Interventionen

\begin{tabular}{lll}
\hline & $\begin{array}{l}\text { Zustimmung } \\
\text { Häufigkeit } \\
\text { (Prozent) }\end{array}$ & $\begin{array}{l}\text { Keine Zustimmung } \\
\text { Häufigkeit } \\
\text { (Prozent) }\end{array}$ \\
\hline 2.1 Ich nutze Körperarbeit bei meinen Coachees & 121 & 112 \\
& $(50,0)$ & $(46,3)$ \\
2.2 Ich praktiziere Körperarbeit/Körperübungen während & 126 & 107 \\
Coachings bei mir selbst (z. B. zur Selbstregulation, Über- & $(52,1)$ & $(44,2)$ \\
tragung, etc.) & & \\
2.3 Ich leite körperbezogene Interventionen bei meinen & 139 & 94 \\
Coachees an & $(57,4)$ & $(38,8)$ \\
2.4 Ich empfehle meinen Coachees Körperübungen & 143 & 90 \\
& $(59,1)$ & $(37,2)$ \\
2.5 Anderes & 25 & 207 \\
& $(10,3)$ & $(85,5)$ \\
3.1 Als Entspannungstechnik für meine Coachees & 135 & 98 \\
& $(55,8)$ & $(40,5)$ \\
3.2 Als konkrete Hilfe/Intervention zur Problembewälti- & 171 & 62 \\
gung oder Zielerreichung meiner Coachees & $(70,7)$ & $(25,6)$ \\
3.3 Als Hilfestellung während den Coachingsitzungen für & 103 & 130 \\
mich persönlich & $(42,6)$ & $(53,7)$ \\
3.4 In meinem Verständnis von Coaching gehört Körperar- & 93 & 140 \\
beit immer dazu (kein besonderes Ziel) & $(38,4)$ & $(57,9)$ \\
3.5 Anderes & 19 & 213 \\
& $(7,9)$ & $(88,0)$ \\
\hline
\end{tabular}

Anmerkung: $N=242$. Abweichungen sind auf fehlende Werte zurückzuführen 
Tab. 6 Andere Ziele beim Einsatz des Körpers im Coaching



Anmerkung: Basierend auf den Antworten von $n=29$ Coaches.

Tab. 7 Wichtigkeit Einbezug Körper im Coaching

$\begin{array}{lllll}\begin{array}{l}\text { Nicht sehr } \\ \text { Häufigkeit } \\ \text { (Prozent) }\end{array} & \begin{array}{l}\text { Teils-teils } \\ \text { Häufigkeit } \\ \text { (Prozent) }\end{array} & \begin{array}{l}\text { Ja } \\ \text { Häufigkeit } \\ \text { (Prozent) }\end{array} & \begin{array}{l}\text { Ja, sehr } \\ \text { Häufigkeit } \\ \text { (Prozent) }\end{array} & \begin{array}{l}\text { Kumulierte } \\ \text { Werte } \\ \text { Klare Zu- } \\ \text { stimmung }\end{array} \\ \text { (Prozent) }\end{array}$

4. Ich finde es wichtig, den Körper im Coaching miteinzubeziehen

5. Erleben Sie Körperarbeit im Coaching als zielführend und/oder gewinnbringend?

\section{Anmerkung:}

a Kumulation der Kategorien ,ja“" und ,ja, sehr“

der Diskussion thematisiert. Mehr als die Hälfte $(52,1 \%)$ praktizieren Körperarbeit während des Coachings auch bei sich selbst.

In einer offenen Frage konnten die Coaches angeben, welche weiteren Ziele sie mit körperorientierten Interventionen verfolgen (Tab. 6). Unter „Anderes“ wurden u.a. als Ziele des Einsatzes von körperorientierten Interventionen die „Selbstzentrierung der Coaches nach einer Sitzung“, das „Entwickeln neuer Möglichkeiten im inneren System und in der Interaktion“" sowie eine „breitere Wahrnehmung“" genannt. Auch wurde es von einer Person als „Alternative zum kognitiven Weg“ aufgeführt. 
Abb. 1 Einsatz körperorientierter Interventionen in der Zukunft. Anmerkung: Basierend auf den Antworten von $n=227$ Coaches

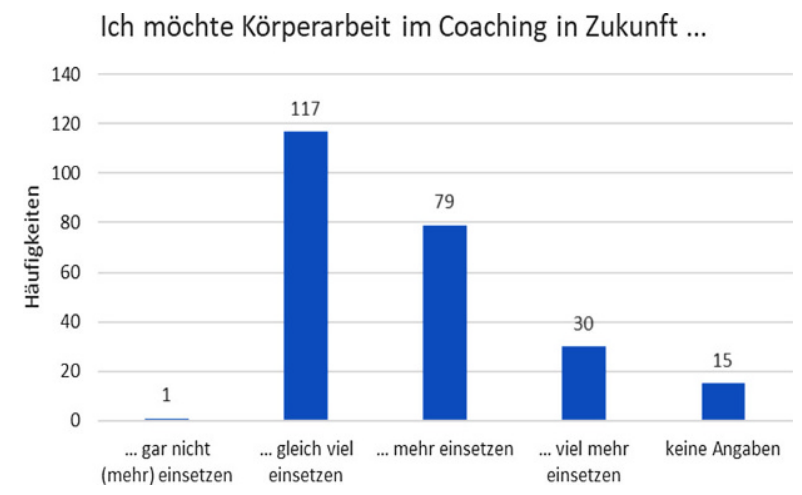

In Tab. 7 sind die Antworten zur Wichtigkeit und Bedeutung von körperorientierten Interventionen dargestellt. Eine Mehrheit von fast $73 \%$ der Coaches finden es wichtig oder sehr wichtig, den Körper im Coaching miteinzubeziehen. $18 \%$ finden es ,teils-teils“ wichtig und knapp $4 \%$,nicht sehr wichtig“. Ebenfalls eine Mehrheit von $68 \%$ empfinden körperorientierte Interventionen als gewinnbringend und/ oder zielführend oder sehr gewinnbringend und/oder zielführend. $21 \%$ geben an, es „teils-teils“ als gewinnbringend zu empfinden, und 3\% ,nicht sehr“.

In Abb. 1 werden die Resultate auf die Frage, inwiefern die befragten Coaches körperorientierte Intervention auch in Zukunft einsetzen wollen, gezeigt. Keiner der Befragten Coaches möchte körperorientierte Arbeit in Zukunft weniger nutzen, fast $50 \%$ möchten sie gleich oft anwenden und $45 \%$ beabsichtigen, solche Methoden vermehrt einzusetzen.

\section{Diskussion}

Ziel der Untersuchung war es, einen Einblick zu erhalten, ob, welche Arten und in welcher Intensität Coaches körperorientierte Interventionen im Coaching einsetzen, wie ihre Haltung dazu ist und inwiefern sie beabsichtigen, solche Interventionen in Zukunft einzusetzen.

Die Resultate sind recht deutlich. Jeweils mehr als die Hälfte der befragten Coaches empfiehlt körperorientierte Interventionen, nutzt und leitet sie innerhalb des Coachings an und betrachtet sie als gewinnbringend und zielführend. Eine sehr große Mehrheit beabsichtigt, solche Interventionen in Zukunft mindestens gleich oft oder sogar noch öfter einzusetzen. Und, dies ist aus unserer Sicht bemerkenswert, ebenfalls mehr als die Hälfte der Coaches wendet körperorientierte Interventionen während des Coachings auch auf sich selbst an. Wiederum eine eindeutige Mehrheit von Coaches setzt ein breites Spektrum von köperorientierten Interventionen ein. Nur eine Minderheit der Coaches tut dies praktisch nicht. In Abhängigkeit zur Intervention nutzen mehr als $70 \%$ bis hin zu fast $100 \%$ der Coaches in irgendeiner Form die Arbeit mit dem Körper, um ihre Coachings zu unterstützen. Beträchtliche Unterschiede sind hingegen in der Häufigkeit der Anwendung unterschiedlicher Arten von 
Interventionen festzustellen. Einige wenden solche Interventionen nur gelegentlich, andere wiederum sehr oft an.

Auf der Basis der vorliegenden Resultate kommen wir zu dem Schluss, dass die Aussage von Ryba (2019) und Pullen (2016), Körperressourcen würden nicht oft berücksichtigt resp. spielten nur eine kleine Rolle, zwar auf die einschlägige Literatur zutreffen mag, für die Praxis aber nicht bestätigt werden kann und daher differenziert werden muss. Coaches setzen häufig körperorientierte Methoden ein, sie unterscheiden sich aber in der Art der Interventionen und der Häufigkeit der Anwendung. Fast alle Coaches achten auf Körperhaltung und Mimik, immer noch sehr große Mehrheiten (>90\%) achten auf die Atmung, spiegeln die Körpersprache und nutzen die Körperwahrnehmung ihrer Coachees zur Interventionsreflexion. Interventionen hingegen, die einen aktiveren und intensiveren Einbezug des Körpers voraussetzen, werden nicht mehr so breit, aber immer noch recht häufig eingesetzt. Anstatt von einer Nichtberücksichtigung des Körpers zu sprechen, möchten wir hier vorschlagen, von unterschiedlichen Ebenen der Integration des Körpers in den Coaching-Dialog zu sprechen. Der Körper, z. B. die Mimik oder die Körperhaltung, wird vom Coach als Erkenntnisquelle genutzt, und die Erkenntnisse werden wieder in den Dialog eingebracht.

Interventionen, die einen spezifischeren Einsatz des Körpers und vermutlich auch mehr Wissen, z. B. bei Übungen zur Stress- oder Emotionsregulation, voraussetzen, werden etwas weniger eingesetzt. Warum dies so ist, darüber können wir nur spekulieren. $\mathrm{Ob}$ es sich die Coaches nicht zutrauen, weil die Kompetenz nicht vorhanden ist, ob sie es für nicht angemessen und/oder für den anwesenden Coachee nicht für zielführend halten, ist unklar. Vielleicht entscheiden sie aber aufgrund der Intuition oder aufgrund einer gemeinsamen Entscheidung mit ihren Coachees, körperorientierte Interventionen einzusetzen oder darauf zu verzichten. Für diese Annahme spricht, dass ,nur“ $38 \%$ der Coaches angaben, dass körperorientierte Arbeit immer in ein Coaching gehört. Wobei hier anzumerken ist, dass diese Zahl auch noch anders interpretiert werden kann. Es ist sehr gut möglich, dass Coaches eine andere Definition von körperorientierten Interventionen haben als die Autor/innen und z. B. die Nutzung des Körpers rein als Erkenntnis- und Reflexionsquelle noch nicht als körperorientierte Intervention gedeutet wird, wie es die Autor/innen tun.

In die gleiche Richtung geht die Feststellung eines Widerspruchs zwischen den Antwortverteilungen zu den Fragen, ob körperorientierte Interventionen generell genutzt, empfohlen und angeleitet werden, und denjenigen nach den konkreten Anwendungen solcher Interventionen. Diese hätten nach Ansicht der Autor/innen mehr oder minder deckungsgleich sein sollen, sie sind es aber bei weitem nicht. Die Zustimmungsraten zur Frage, ob die Coaches körperorientierte Interventionen anleiten, beträgt $57 \%$. Die Angaben der Coaches, ob sie eine bestimmte Intervention manchmal oder häufiger ausführen, liegen zwischen 71 und fast $100 \%$. Auch hier erklären wir uns den Unterschied mit den unterschiedlichen, evtl. sehr individuellen Definitionen der antwortenden Coaches und der von uns hinterlegten Definition körperorientierter Interventionen, aufgrund derer wir die konkreten Fragen gestalteten. Diese wurde zwar offengelegt; ob man sich bei der Beantwortung der Fragen aber noch darin erinnern konnte, ist zu bezweifeln. In diesem Antwortverhalten spiegelt sich vermutlich nicht nur eine unterschiedliche Sichtweise der Antwortenden und 
der Autor/innen dieses Beitrags, sondern auch ein generell sehr unterschiedliches Verständnis des Begriffs „,körperorientierte Intervention“.

Methodenkritik Obwohl die Stichprobe hinsichtlich der Anzahl der Teilnehmenden, den Erfahrungsjahren als Coach, dem Beschäftigungsgrad und der Ausbildung anderen Coachingumfragen entspricht (Gross 2012; Rauen 2020), Kennwerte zur Grundgesamtheit aber fehlen, ist es unklar, ob die Resultate für die DACH-Region - o der auch nur für die deutschsprachige Schweiz - repräsentativ sind. Dagegen sprechen das durchschnittliche Alter der Coaches (dieses ist mit 53 Jahren ein wenig höher als in vergleichbaren Umfragen) und die Tatsache, dass weibliche Coaches mit $71 \%$ im Vergleich zu anderen Coachingerhebungen (Jonassen et al. 2009; Seiger und Künzli 2011, 2012; Hofmann und Künzli 2021; Rauen 2020) übervertreten sind. Übervertreten sind auch Absolvent/innen des Instituts für Angewandte Psychologie IAP. Immerhin ein gutes Viertel der Stichprobe hat irgendeine Form einer körperorientierten Ausbildung absolviert. Ob dies die tatsächlichen Verhältnisse in der Grundgesamtheit spiegelt oder ob es sich um eine den körperorientierten Interventionen zu- oder allenfalls abgeneigte Stichprobe handelt, kann hier nicht beantwortet werden, da uns hierzu keine Vergleichsdaten bekannt sind.

Zukünftige Forschung Die Umfrage gewährt einen ersten Blick in den Stand der Dinge körperorientierter Interventionen im Coaching. Von einem systematischen Wissen darüber, ob, wann und wie diese Interventionen eingesetzt werden sollen, sind wir aber noch weit entfernt. Insbesondere wissen wir wenig bis nichts:

- über den angemessenen Einsatz von körperorientierten Interventionen im Coaching. Ist deren Einsatz einfach „Geschmackssache“, individuelle Vorliebe, oder gibt es klare Indikationen,

- über die Wirkung von körperorientierten Methoden im Coaching. Gibt es Interventionen der Wahl? Sind Coachings, in denen körperorientierte Interventionen eingesetzt werden, im Vergleich zu stark sprachbasierten Vorgehensweisen überlegen, unterlegen oder gleichwertig? Oder gilt auch hier eine Form des DodoVerdikts, dass das Vorgehen keine Rolle spielt, solange ein in sich kohärentes Set von Regeln, wie z.B. allgemeine Wirkfaktoren, angemessen angewendet wird,

- über die tatsächliche Verbreitung von körperorientierten Interventionen in der Coaching-Praxis. Sind die aus Sicht der Autor/innen überraschend hohe Werte der Verbreitung körperorientierter Interventionen im Coaching tatsächlich repräsentativ,

- darüber, inwiefern Coach-Merkmale, wie z. B. Alter, Geschlecht oder Erfahrungsjahre, die Präferenzen in Hinsicht auf körperorientierte Interventionen beeinflussen,

- darüber, welche Inhalte, welche Kompetenzen in Hinsicht auf den Körper mindestens zu fordern sind für eine Coaching-Ausbildung resp. für eine Vertiefung in körperorientiertem Coaching,

- darüber, welche Ausbildungsinstitute in welchem Ausmaß schon heute körperorientierte Methoden vermitteln. 
Ausblick Vertraut man den Daten, dann ist Körperarbeit im Coaching schon recht gut verankert und wird in Zukunft noch an Bedeutung gewinnen. Klare Mehrheiten beschreiben die Körperarbeit als wichtig, erleben sie als zielführend, und $45 \%$ der Befragten wollen in Zukunft sogar vermehrt Körperarbeit einsetzen. Dies ist, um hier eine subjektive Wertung zu wagen, aus Sicht der Autor/innen durchaus erfreulich, da wir beide körperorientierten Methoden positiv gegenüberstehen. Den Artikel mit dieser leicht rosa gefärbten Aussage zu schließen, wäre aber ein wenig naiv.

Die Coaching-Landschaft entwickelt sich sehr dynamisch. Wenn man sich vor Augen hält, wie schnell sich allein der Begriff entwickelte, in welchen Zeitabständen neue Angebote aus dem Boden schießen, dann darf man vermuten, dass sich körperorientierte Angebote, sofern der Markt vorhanden ist, im Coaching sehr schnell (weiter-) entwickeln werden. Diese Entwicklung beinhaltet aber auch Gefahren. Es gibt keine Interventionen ohne Risiken und Nebenwirkungen; was wirkt, kann auch schaden, und der „normale“ Coach hat keine medizinische Ausbildung und ist kein Experte für den Körper. Einfach zu fordern, vermehrt körperorientierte Methoden einzusetzen, weil dies aus einer naiven Position ,ganzheitlicher“ wäre, ist sicher nicht vertretbar. Hier sind die Verbände und die Ausbildungsinstitute gefordert, Richtlinien zu erlassen und Weiterbildungsinhalte $\mathrm{zu}$ entwickeln, die der Tatsache Rechnung tragen, dass potenzielle und praktizierende Coaches in der Regel über wenig oder gar kein professionelles Vorwissen in Hinsicht auf den Körper verfügen. Wie weiter oben schon angesprochen, geht es nicht um die Frage, ob körperorientierte Intervention eingesetzt werden (sollen), sondern viel mehr um das „Wann“, das „Wie“" und die Ebene, auf der der Körper in die Arbeit integriert werden soll. Von der Wahrnehmung und Rückspiegelung der Körperhaltung über sanfte Entspannungsübungen bis hin zu Methoden, die starke Kontrollaufgabe auf Seiten der Coachees voraussetzen, ist es ein weiter Weg. Wie in der Medizin sollte auch im Coaching der Grundsatz „Primum non nocere“, man solle vor allem nicht schaden, gelten, was voraussetzt, dass Coaches zu jeder Zeit im Prozess wissen sollten was sie tun.

Funding Open access funding provided by ZHAW Zurich University of Applied Sciences

Open Access Dieser Artikel wird unter der Creative Commons Namensnennung 4.0 International Lizenz veröffentlicht, welche die Nutzung, Vervielfältigung, Bearbeitung, Verbreitung und Wiedergabe in jeglichem Medium und Format erlaubt, sofern Sie den/die ursprünglichen Autor(en) und die Quelle ordnungsgemäß nennen, einen Link zur Creative Commons Lizenz beifügen und angeben, ob Änderungen vorgenommen wurden.

Die in diesem Artikel enthaltenen Bilder und sonstiges Drittmaterial unterliegen ebenfalls der genannten Creative Commons Lizenz, sofern sich aus der Abbildungslegende nichts anderes ergibt. Sofern das betreffende Material nicht unter der genannten Creative Commons Lizenz steht und die betreffende Handlung nicht nach gesetzlichen Vorschriften erlaubt ist, ist für die oben aufgeführten Weiterverwendungen des Materials die Einwilligung des jeweiligen Rechteinhabers einzuholen.

Weitere Details zur Lizenz entnehmen Sie bitte der Lizenzinformation auf http://creativecommons.org/ licenses/by/4.0/deed.de. 


\section{Literatur}

bso (2019). Institute mit bso-anerkannten Lehrgängen, Institute mit vertraglichen Ausbildungspartnerschaften und Kollektivmitglieder. https://www.bso.ch/fileadmin/user_upload/03_Aus_Weiterbildung/ bso_anerkannte_Ausbildung/PDF_s/Ausbildungsadressen_bso_Juni2017.pdf. Zugegriffen: 30. Nov. 2020.

Geuter, U. (2019). Praxis Körperpsychotherapie. 10 Prinzipien der Arbeit im therapeutischen Prozess. Heidelberg: Springer.

Gross, P.-P. (2012). Eine ökonomische Analyse der wissensintensiven Dienstleistung Coaching. Dissertation. http://archiv.ub.uni-marburg.de/diss/z2013/0408/pdf/dpg.pdf. Zugegriffen: 7. Jan. 2021.

Hofmann, S., \& Künzli, H. (2021). Schweizerischer Coachingmarkt aus Sicht der Coaches. Winterthur: Zürcher Hochschule für Angewandte Wissenschaften (im Druck).

Jonassen, M., Seiger, C., \& Künzli, H. (2009). Der Coachingmarkt in der Deutschschweiz. Winterthur: Zürcher Hochschule für Angewandte Wissenschaften.

Mayring, P. (2010). Qualitative Inhaltsanalyse: Grundlagen und Techniken. Weinheim: Beltz.

Pesendorfer, D., \& Pechtl, C. (2018). Praxis körperbezogener Beratung. Supervision, 2018(3), 35-40.

Pullen, J. C. (2016). Der Körper als Ressource in individuellen Veränderungsprozessen. Organisationsberatung, Supervision, Coaching, 23(3), 285-296.

Rauen, C. (2020). RAUEN Coaching-Marktanalyse 2020. Version vom 03.09.2020. https://www.rauen.de/ cma/. Zugegriffen: 30. Nov. 2020.

Rolef Ben-Shahar, A. (2014). Touching the relational edge. Body psychotherapy. London: Karnac.

Ryba, A. (2019). Körperzentrierte Coaching und der Zugang zum Unbewussten. Organisationsberatung, Supervision, Coaching, 26(3), 313-329. https://doi.org/10.1007/s11613-019-00610-1.

Ryba, A., \& Roth, G. (Hrsg.). (2019). Coaching und Beratung in der Praxis. Ein neurowissenschaftlich fundiertes Integrationsmodell. Stuttgart: Klett-Cotta.

Schatz, D.S. (2002). Klassifikation des Körpererlebens und körperpsychotherapeutische Hauptströmungen. Psychotherapeut, 47, 77-82.

Schley, M. (2015). Bewegung und Therapie aus leibphänomenologischem und konsistenztheoretischem Blickwinkel. Körper - tanz - bewegung, 3, 144-150.

Seiger, C., \& Künzli, H. (2011). Schweizerischer Coachingmarkt aus Sicht der Coachs. Winterthur: Zürcher Hochschule für Angewandte Wissenschaften.

Seiger, C., \& Künzli, H. (2012). Schweizerischer Coachingmarkt aus Sicht der Coachs. Winterthur: Zürcher Hochschule für Angewandte Wissenschaften.

Storch, M., Cantieni, B., Hüther, G., \& Tschacher, W. (2017). Embodiment. Die Wechselwirkung von Körper und Psyche verstehen und nutzen. Bern: Hogrefe.

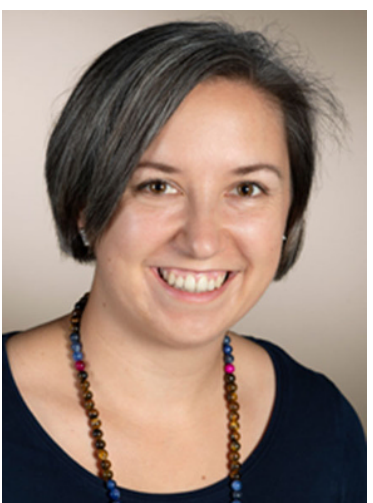

Natascha Lienhard BSc in Angewandter Psychologie, geb. 1989, ist wissenschaftliche Assistentin in der Fachgruppe Diagnostik und Beratung am Departement Psychologie der Zürcher Hochschule für Angewandte Wissenschaften. Sie schreibt zurzeit an ihrer Masterarbeit zum Thema „Achtsamkeitsbasierte Stressprävention via Chatbot bei Studierenden". 


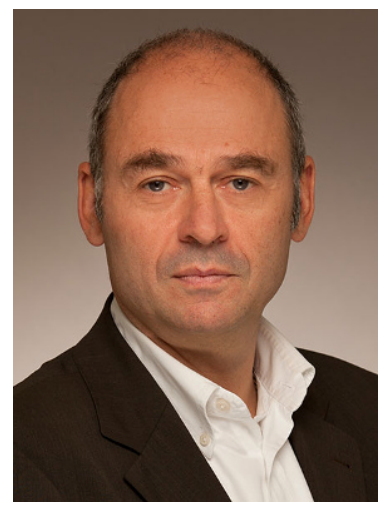

Prof. Hansjörg Künzli geb. 1962, leitet die Fachgruppe Diagnostik und Beratung am Departement Psychologie der Zürcher Hochschule für Angewandte Wissenschaften. Forschungs- und Lehrtätigkeit in den Bereichen Coaching, Beratung, Chatbotcoaching und Testentwicklung. Ausbildungen in systemisch-lösungsorientierter Organisationsentwicklung und hypnosystemischem Coaching. 